\title{
Multicenter, Observational Study of Lanreotide Autogel for the Treatment of Patients with Neuroendocrine Tumors in Routine Clinical Practice in Germany and Austria
}

\section{(ㄷ)(1) (우 $\Theta$}

\author{
Authors \\ Anja Rinke', Christoph Maintz² ${ }^{2}$ Lothar Müller ${ }^{3}$, Matthias M. Weber ${ }^{4}$, Harald Lahner ${ }^{5}$, Marianne Pavel ${ }^{6}$, \\ Wolfgang Saeger ${ }^{7}$, Aude Houchard ${ }^{8}$, Hanna Ungewiss ${ }^{9}$, Stephan Petersenn ${ }^{10}$
}

\author{
Affiliations \\ 1 Department of Gastroenterology and Endocrinology, \\ University Hospital Gießen and Marburg, Marburg, Germany \\ 2 Hämatologisch-Onkologische Praxis, MVZ West GmbH, \\ Würselen, Germany \\ 3 Onkologie Unter-Ems, Leer, Germany \\ 4 Unit of Endocrinology, Department of Medicine 1, \\ University Medical Center of the Johannes Gutenberg- \\ University Mainz, Mainz, Germany \\ 5 Department of Endocrinology, Diabetes and Metabolism, \\ Division of Laboratory Research, University Hospital Essen, \\ Germany \\ 6 Department of Medicine 1, Division of Endocrinology, \\ Universitätsklinikum Erlangen, Erlangen, Germany \\ 7 Department of Neuropathology, Pituitary Pathologist, \\ University Hospital Eppendorf, Hamburg, Germany \\ 8 Ipsen, Boulogne Billancourt, France \\ 9 Ipsen, München, Germany \\ 10 ENDOC Center for Endocrine Tumors, Hamburg, Germany
}

Key words

chromogranin A, carcinoid syndrome, diarrhea, flushing, quality of life

received 18.09 .2020

revised 07.12 .2020

accepted 22.12 .2020

Bibliography

Exp Clin Endocrinol Diabetes 2021; 129: 500-509

DOI 10.1055/a-1342-2755

ISSN $0947-7349$

(C) 2021. The Author(s).

This is an open access article published by Thieme under the terms of the Creative Commons Attribution-NonDerivative-NonCommercial-License, permitting copying and reproduction so long as the original work is given appropriate credit. Contents may not be used for commercial purposes, or adapted, remixed, transformed or built upon. (https://creativecommons. org/licenses/by-nc-nd/4.0/)

Georg Thieme Verlag KG, Rüdigerstraße 14,

70469 Stuttgart, Germany

\author{
Correspondence \\ Prof. Dr. med. Stephan Petersenn \\ ENDOC Center for Endocrine Tumors \\ Erik-Blumenfeld-Platz 27A \\ 22587 Hamburg \\ Germany \\ Tel.: + 4940401 87985, Fax: +494040186629 \\ stephan.petersenn@endoc-med.de
}

\section{ABSTRACT}

Background The long-acting somatostatin analog lanreotide autogel is effective in the treatment of patients with neuroendocrine tumors.

Objective To evaluate the long-term treatment response in patients with neuroendocrine tumors receiving lanreotide autogel in routine clinical practice.

Methods Non-interventional, 24-month study in patients with neuroendocrine tumors treated with lanreotide autogel (NCT01840449).

Results Patients ( $\mathrm{n}=80$ ) from 26 centers in Germany and Austria were enrolled. Neuroendocrine tumors were mainly grade $1 / 2$, metastasized, intestinal, and associated with carcinoid syndrome; $88.9 \%$ had received previous neuroendocrine tumor treatment. Of those, $84.4 \%$ had previous surgery, $18.7 \%$ had received octreotide. The primary endpoint, defined by a $<50 \%$ chromogranin A increase at month 12 compared with the lowest value between baseline and month 3 was achieved by $89.5 \%$ patients. Stable disease according to Response Evaluation Criteria in Solid Tumors 1.1 was observed in 76.9 and $75.0 \%$ patients at months 12 and 24 of lanreotide treatment, respectively. Mean change of chromogranin A levels from baseline to month 24 was $-0.12 \times$ upper limit of normal $(95 \% \mathrm{Cl},-0.22 ;-0.45)$. In a post hoc analysis, $38.5 \%$ of the subgroup of patients with carcinoid syndrome had daily diarrhea at baseline vs. $21.4 \%$ at month 24 . At baseline, $27.8 \%$ of patients received lanreotide $120 \mathrm{mg}$ every 4 weeks vs. $56.7 \%$ at month 24 . Quality of life data were heterogeneous. No new safety issues arose and/or required further investigation.

Conclusions Our study reflects routine lanreotide autogel use in patients with advanced/metastatic neuroendocrine tumors. This analysis shows effectiveness with stabilization of disease-related symptoms and good tolerability of lanreotide autogel in clinical practice. 


\section{Introduction}

Neuroendocrine tumors (NET) are rare tumors usually localized in the gastrointestinal tract, lung, and pancreas [1]. They have a low, but increasingly reported, incidence of up to 7/100000 [1]. Most patients present with advanced, unresectable disease due to local extension or metastasis. Treatment options for gastroenteropancreatic (GEP) NET include curative resection of the primary tumor and metastases, and systemic therapies to control tumor growth and alleviate hormonal syndromes. In addition, treatment aims to maintain or improve patients' quality of life [2]. Prognosis depends on primary tumor location, presence of metastasis in the liver and extrahepatic tissues, proliferative activity (tumor grade) and functional activity [1].

Advanced unresectable low-grade NETs are usually treated by antiproliferative drugs such as somatostatin analogs. Upon progression, peptide receptor radionuclide therapy (PRRT) or targeted therapies as well as loco-regional treatments represent further therapy options; in pancreatic NET systemic chemotherapy (Streptozocin or temozolomide based) is another established therapy [3]. In high-grade neuroendocrine carcinomas (NECs) cytotoxic chemotherapy with cisplatin and etoposide is first line therapy [3].

Approximately $30-40 \%$ of patients with well-differentiated NET present with carcinoid syndrome due to excessive production of serotonin and other bioactive compounds by the tumor [4]. Hallmarks of disease are diarrhea and flushing. In these patients, somatostatin analogs are a choice for antisecretory treatment [3]. A number of randomized, controlled and observational studies have shown that the long-acting somatostatin analog lanreotide autogel (LAN) effectively controls functional activity and symptoms, particular in serotonin-secreting NETs [5-9].

In somatostatin receptor (SSTR) positive non-functional NET grade 1 (G1) with low tumor burden both, somatostatin analogs or 'watch and wait' are considered as clinical options. Furthermore, somatostatin analogs are indicated for first-line treatment to control tumor growth of patients with SSTR positive, low to intermediate proliferative (Ki-67 $\leq 10 \%$ ) NET G2 with or without high tumor burden or with progressive disease or symptoms [3]. However, some experts consider a lower Ki-67 cut-off (e.g., $5 \%$ ) a more appropriate threshold to choose more aggressive treatment options [3]. Anti-tumor effects for LAN were confirmed in the randomized controlled 96-week CLARINET trial $[10,11]$. LAN prolonged progression-free survival over placebo in patients with metastatic $\mathrm{G} 1$ or G2 (proliferation index, Ki-67 $\leq 10 \%$ ) SSTR positive NET of pancreatic, intestinal or of unknown primary origins. Most patients had prior stable disease (RECIST 1.0), and efficacy of LAN was observed irrespective of hepatic tumor volume and grade [10,11]. LAN is currently licensed in Europe for the treatment of grade 1 (G1) and a subset of grade 2 (Ki-67 index $\leq 10 \%$ ) NETs of midgut, pancreatic or unknown origin, in adult patients with unresectable locally advanced or metastatic disease [12], and in the US for the treatment of patients with unresectable, well-or moderately-differentiated, locally advanced or metastatic GEP-NETs to improve progression-free survival [13].

This non-interventional study was designed to evaluate the long-term response to LAN treatment in patients with NET in routine clinical practice, and to investigate factors that might corre- late with treatment success. The secondary objective was to better understand the patient population receiving treatment with LAN for NET in Germany and Austria.

\section{Methods}

\section{Patients}

Adult patients aged 18 years and older diagnosed with functional or non-functional NET to be treated de novo with LAN or who had previously been on treatment with LAN for less than 6 months (maximum one-third of the enrolled patients allowed) could be included. In patients previously treated with LAN, baseline data before starting LAN treatment and all other available data since baseline were assessed retrospectively. Prospective documentation followed from the time of inclusion. Patients were recruited from medical practices or clinics in Germany and Austria with special expertise in the treatment of patients with NET. Target enrolment was 76 patients. Written informed consent was obtained from all patients prior to inclusion. The efficacy population comprised all enrolled patients for whom core data (age, gender, date of baseline, date of LAN administration at baseline, dose of LAN administration at baseline and diagnosis of NET) were collected in the electronic case report form; who did not start LAN treatment more than 30 days prior to baseline; and who had a chromogranin $A(C g A)$ value from at least two time points including baseline and/or month 3 , and months 3, 6, 12, 18 and/or month 24.

\section{Study design}

This was a multicenter, non-interventional, observational study in Germany and Austria. Both prospective and retrospective (maximum one-third of the enrolled patients) documentation was allowed. The patients received treatment as prescribed by the investigator and in accordance with routine practice. All diagnostic and therapeutic decisions were in the hands of the treating physician and completely independent of the decision to include the patient in this study. Routine visits were recorded at baseline and at approximately $1,3,6,12,18$ and 24 months.

This study was conducted in compliance with independent ethics committees/institutional review boards, informed consent regulations, the Declaration of Helsinki (Version 2013) and International Conference on Harmonisation (ICH) Good Clinical Practice (GCP) Guidelines.

\section{Endpoints}

The primary endpoint was the long-term response, i. e. the control rate after 1 year, defined by $\mathrm{CgA}$ level $<50 \%$ increase compared with lowest $\mathrm{CgA}$ level between baseline and month 3. Secondary endpoints included changes of symptoms, global evaluation of effectiveness by the physician, global evaluation of tolerability by the patients, and quality of life (QoL).

\section{Assessments}

Effectiveness parameters (all assessed as per routine practice) included basal $\mathrm{CgA}$ serum levels, 24-hour urinary excretion of 5-hydroxyindoleacetic acid (5-HIAA) in patients with carcinoid syn- 
drome and clinical parameters such as presence and frequency of diarrhea and flushing. Additional laboratory assessments included fasting blood glucose, and glycosylated hemoglobin $\left.\left[\mathrm{HbA}_{1 \mathrm{c}}\right]\right)$. All laboratory parameters were tested by local laboratories. Response Evaluation Criteria In Solid Tumors (RECIST) 1.1 was used to assess tumor response, the Eastern Co-operative Oncology Group (ECOG) scale was used to evaluate the performance status. Global evaluation of overall effectiveness was subjectively rated by the investigator by categories (very poor, poor, moderate, good, and very good). Quality of life (QoL) was assessed by the German validated version of the European Organization for Research and Treatment of Cancer (EORTC) Study Group's 30-item Quality of Life Questionnaire (QLQ-C30) (subscores: global health status; physical, role, emotional, cognitive and social functional scales and symptom scales) and, in addition, by the disease-specific 21-item EORTC QLQ-GINET21 questionnaire (subscores: treatment-related symptoms, weight gain, information/communication function, sexual function, endocrine symptoms, gastrointestinal symptoms, social function, disease-related worries, muscle/bone pain symptoms and body image). For health-related quality of life scores a change of 10 -points is frequently considered a minimal clinically important difference $[14,15]$. If routinely performed, quality of life was assessed in the patients at baseline and at approximately 1, 6, 12, 18 and 24 months.

If available, tumor tissue from previous surgery could be submitted optionally for analysis of SSTR status (subtypes 2, 3, and 5). SSTR analyses were all performed by immunohistochemistry (IHC) at the Department of Neuropathology, University Hospital Eppendorf, Hamburg, Germany. For immunohistochemistry polyclonal rabbit anti-SSTR2A, rabbit anti-SSTR5 (both Zytomed Systems, Bargteheide, Germany) and rabbit anti-SSTR3 (Thermo Fisher, Rockford, USA) antibodies were used.

Adverse event (AE) reporting followed regulations related to non-interventional post-authorization studies. Any non-serious or serious $A E s$ related to the product as well as any serious $A E$, independently of the relationship to the product, were reported to the clinical research organization and pharmacovigilance. Unrelated non-serious AEs were not collected routinely, as they were not considered likely to add relevant new information on the safety of the product. The presence of gallbladder stones was assessed by ultrasound as per clinical practice. Global evaluation of tolerability was assessed by the patient by categories (very poor, poor, moderate, good, and very good).

\section{Statistical analysis}

All analyses are descriptive. Data are presented as mean and $95 \%$ confidence interval $(\mathrm{Cl})$ or as median and range. The primary analysis was performed on the efficacy population (see above). In addition, post hoc analyses were performed for biochemical response, symptoms and SSTR status in the subpopulation of patients with carcinoid syndrome. SSTR expression based on scintigraphy results were presented according to location and functional activity of the primary tumor.

\section{Results}

\section{Patient disposition}

Patients with NET ( $n=80$ ) were enrolled from 26 medical practices and clinics, of whom 25 patients were documented retrospectively. Each site contributed between one and eleven patients. 48 patients continued the study until month 12, 31 patients continued until month 24. Reasons for discontinuation until month $24(n=48)$ were decreasing efficacy of treatment $(n=13)$, loss to follow-up $(n=12)$, AEs $(n=6)$, withdrawal of consent $(n=2)$, death $(n=9)$, and other reasons $(n=6)$. The efficacy population consisted of 42 patients. Of these, 20 patients discontinued prematurely before month 24 due to decreasing efficacy of treatment $(n=8)$, loss to follow-up $(n=4)$, death $(n=4)$, and other reasons $(n=4) .22$ patients of the efficacy population completed the study.

\section{Patient characteristics}

- Table 1 shows the baseline characteristics of the enrolled and efficacy populations. Mean age of enrolled patients was 65.7 years (95\% Cl, 62.9-68.5), 54.4\% were male. Median disease duration was 1.5 years $(95 \% \mathrm{Cl}, 0.5-2.6)$ after symptom onset. Of the enrolled patients, 50 (63.3\%) NETs were intestinal. Of these, 35 tumors were located in the ileum, four in the stomach, three were located in each, duodenum and jejunum, five in the caecum, and one in each appendix, colon and rectum. $24.1 \%$ of tumors were located in the pancreas and $22.8 \%$ were located in other sites. In $69.6 \%$ of patients, NETs were classified as functioning, in particular as carcinoid syndrome in $64.6 \%$ of enrolled patients. Two patients had an insulinoma, and one patient a gastrinoma or somatostatinoma each. Non-functioning NETs were present in $30.0 \%$ of patients. $88.6 \%$ of patients had metastatic disease, mainly localized in the liver (72.2\%) and in the lymph nodes (62.0\%). Of the tumors, $53.8 \%$ were $\mathrm{G} 1,41.0 \% \mathrm{G} 2,5.1 \% \mathrm{G} 3$. Of the patients, $88.9 \%$ had received previous treatment for NET. Of these pre-treated patients, $84.4 \%$ had previous surgery and $18.7 \%$ had been medically treated with octreotide LAR. Few patients had been treated with other options such as PRRT, chemotherapy, targeted therapy, local ablative procedures, or interferon. Most frequent comorbidities at baseline were diabetes mellitus (16.5\%) and heart failure (8.9\%).

NET tissue samples to study SSTR expression by IHC were collected for 17 patients. Of these, $94.1 \%$ expressed SSTR2/2a, $52.9 \%$ SSTR5 and $17.6 \%$ SSTR3. SSTR2/2a-positive patients mainly showed high $(+++, 64.7 \%$ ) levels of receptor expression (>Fig. 1$)$. In the patients with carcinoid syndrome and available SSTR status ( $n=13)$, expression of SSTR2/2a was present in $92.3 \%$ patients, SSTR5 in $53.8 \%$, and SSTR3 in $23.1 \%$ patients.

$80 \%$ of patients with NET and carcinoid syndrome with SSTR status available were SSTR-positive according to SSTR scintigraphy. This proportion of patients was similar to those with other NET subtypes (78.9\%). Similar results were observed by location (intestinal: $71.4 \%$, pancreatic: $81.8 \%$, other: $71.4 \%$ ) and grading (NET G1: $78.1 \%$, NET G2: $77.8 \%$, NET G3: $100 \%)$.

\section{Lanreotide dose}

At the baseline visit $45.6 \%$ of patients were administered LAN at a starting dose of $60 \mathrm{mg}, 26.6 \%$ at a dose of $90 \mathrm{mg}$ and $27.8 \%$ at a 
- Table 1 Baseline characteristics of the patients.

\begin{tabular}{|c|c|c|c|c|}
\hline \multirow[t]{2}{*}{ Variable } & \multicolumn{2}{|c|}{ Enrolled population * } & \multicolumn{2}{|c|}{ Efficacy population } \\
\hline & $\mathbf{N}$ & & $\mathbf{N}$ & \\
\hline Age, mean (95\% Cl) & 79 & $65.7(62.9-68.5)$ & 42 & $65.6(61.8-69.4)$ \\
\hline Female/male, n (\%) & 79 & $36(45.6) / 43(54.4)$ & 42 & $19(45.2) / 23(54.8)$ \\
\hline $\mathrm{BMI}, \mathrm{kg} / \mathrm{m}^{2}$, mean $(95 \% \mathrm{Cl})$ & 68 & $25.2(24.1-26.2)$ & 37 & $25.3(23.7-26.8)$ \\
\hline Duration of NET, median yrs $(95 \% \mathrm{Cl})$ & 79 & $1.5(0.5-2.6)$ & 42 & $1.7(0.5-2.6)$ \\
\hline Duration of symptoms, median yrs $(95 \% \mathrm{Cl})$ & 79 & $1.8(0.8-2.6)$ & 42 & $1.8(0.7-4.2)$ \\
\hline NET localizations, $\mathrm{n}(\%) *$ & 79 & & 42 & \\
\hline Intestinal & & $50(63.3)$ & & $29(69.0)$ \\
\hline Stomach & & $4(5.1)$ & & $2(4.8)$ \\
\hline Duodenum & & $3(3.8)$ & & $1(2.4)$ \\
\hline Jejunum & & $3(3.8)$ & & $1(2.4)$ \\
\hline Ileum & & $35(44.3)$ & & $22(52.4)$ \\
\hline Appendix & & $1(1.3)$ & & $1(2.4)$ \\
\hline Caecum & & $5(6.3)$ & & $2(4.8)$ \\
\hline Colon & & $1(1.3)$ & & $1(2.4)$ \\
\hline Rectum & & $1(1.3)$ & & $0(0.0)$ \\
\hline Pancreas & & $19(24.1)$ & & $11(26.2)$ \\
\hline Other & & $18(22.8)$ & & $6(14.3)$ \\
\hline Classification, n (\%) & 79 & & 42 & \\
\hline Carcinoid syndrome & & $51(64.6)$ & & $26(61.9)$ \\
\hline Insulinoma & & $2(2.5)$ & & $2(4.8)$ \\
\hline Gastrinoma & & $1(1.2)$ & & $0(0.0)$ \\
\hline Somatostatinoma & & $1(1.2)$ & & $0(0.0)$ \\
\hline Other (non-functioning) & & $24(30.0)$ & & $14(33.3)$ \\
\hline Metastases, n (\%) & 79 & & 42 & \\
\hline Any & & $70(88.6)$ & & $38(90.5)$ \\
\hline Liver & & $57(72.2)$ & & $29(69.0)$ \\
\hline Lymph nodes & & $49(62.0)$ & & $28(66.7)$ \\
\hline Lung & & $6(7.6)$ & & $2(4.8)$ \\
\hline Other & & $26(32.9)$ & & $12(28.6)$ \\
\hline Grading, n (\%) & 78 & & 42 & \\
\hline G1 & & $42(53.8)$ & & $23(54.8)$ \\
\hline G2 & & $32(41.0)$ & & $16(38.1)$ \\
\hline G3 & & $4(5.1)$ & & $3(7.1)$ \\
\hline \multicolumn{5}{|l|}{$\begin{array}{l}\text { Positive SSTR scintigraphy, n (\%) } \\
\text { By type }\end{array}$} \\
\hline Carcinoid syndrome & 35 & $28(80.0)$ & 21 & $17(81.0)$ \\
\hline Other & 19 & $15(78.9)$ & 12 & $9(75.0)$ \\
\hline \multicolumn{5}{|l|}{ By localization } \\
\hline Intestinal & 32 & $25(78.1)$ & 21 & $16(76.2)$ \\
\hline Pancreas & 11 & $9(81.8)$ & 8 & $6(75.0)$ \\
\hline Other & 7 & $5(71.4)$ & 2 & $2(100.0)$ \\
\hline \multicolumn{5}{|l|}{ By grade } \\
\hline G1 & 32 & $25(78.1)$ & 19 & $14(73.7)$ \\
\hline G2 & 18 & $14(77.8)$ & 11 & $9(81.8)$ \\
\hline G3 & 4 & $4(100.0)$ & 3 & $3(100.0)$ \\
\hline \multicolumn{5}{|l|}{ Previous treatments, $\mathrm{n} / \mathrm{N}(\%){ }^{*}$} \\
\hline Any & 72 & $64(88.9)$ & 37 & $34(91.9)$ \\
\hline Surgery & 64 & $54(84.4)$ & 34 & $32(94.1)$ \\
\hline Octreotide LAR & 64 & $12(18.7)$ & 34 & $8(23.5)$ \\
\hline PRRT & 64 & $7(10.9)$ & 34 & $4(11.8)$ \\
\hline
\end{tabular}


Table 1 Continued.

\begin{tabular}{|c|c|c|c|c|}
\hline \multirow[t]{2}{*}{ Variable } & \multicolumn{2}{|c|}{ Enrolled population * } & \multicolumn{2}{|c|}{ Efficacy population } \\
\hline & $\mathbf{N}$ & & $\mathbf{N}$ & \\
\hline Chemotherapy & 64 & $6(9.4)$ & 34 & $2(5.9)$ \\
\hline Targeted therapy & 64 & $6(9.4)$ & 34 & $3(8.8)$ \\
\hline Local ablative procedures & 64 & $4(6.2)$ & 34 & $2(5.9)$ \\
\hline Interferon & 64 & $1(1.6)$ & 34 & $1(2.9)$ \\
\hline Concomitant diseases, $\mathrm{n} / \mathrm{N}(\%){ }^{*}$ & 79 & & 42 & \\
\hline Any & & $20 / 79(25.3)$ & & $9(21.4)$ \\
\hline Diabetes & & $13 / 79(16.5)$ & & $4(9.5)$ \\
\hline Heart failure & & $7 / 79(8.9)$ & & $4(9.5)$ \\
\hline Impaired glucose tolerance & & $1 / 79(1.3)$ & & $1(2.4)$ \\
\hline ECOG status, n (\%) & 78 & & 42 & \\
\hline 0 (fully active) & & $39(50.0)$ & & $20(47.6)$ \\
\hline 1 (restricted activity) & & $31(39.7)$ & & $18(42.9)$ \\
\hline 2 (no work activities) & & $6(7.7)$ & & $4(9.5)$ \\
\hline 3 (only limited self-care) & & $2(2.6)$ & & $0(0.0)$ \\
\hline 4 (completely disabled) & & $0(0.0)$ & & $0(0.0)$ \\
\hline
\end{tabular}

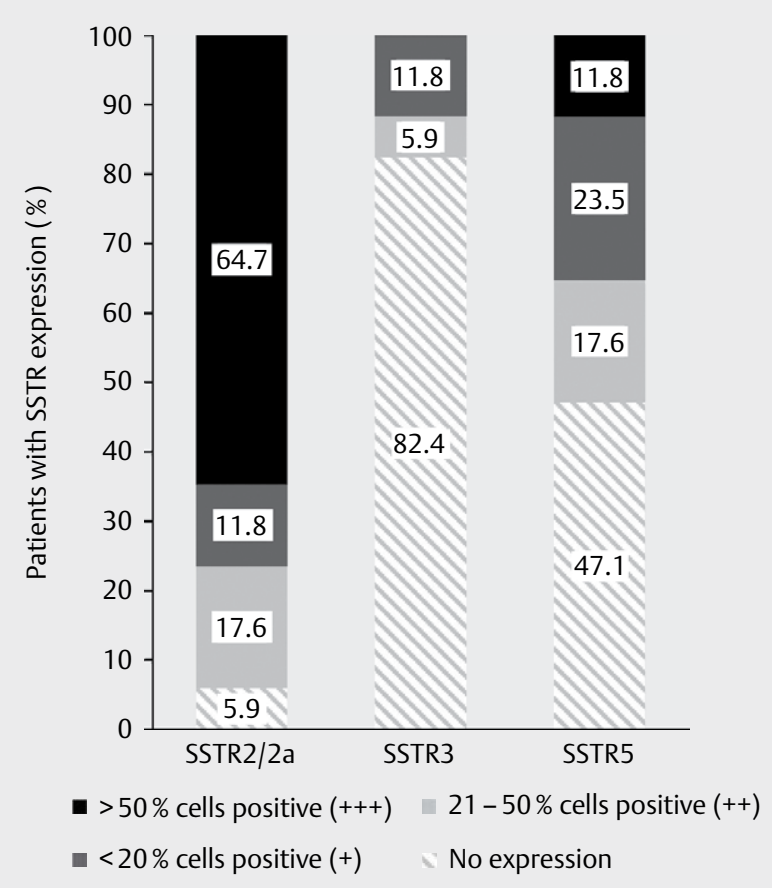

-Fig. 1 Expression of SSTR subtypes at baseline $(n=17)$ (enrolled population); SSTR: somatostatin receptor.

dose of $120 \mathrm{mg}$ every 4 weeks ( $\triangleright$ Fig. 2). The proportion of enrolled patients receiving the $120 \mathrm{mg}$ dose every 4 weeks was $53.3 \%$ at 12 months and $56.7 \%$ at 24 months.

Of patients with carcinoid syndrome $49.0 \%$ were administered LAN at a starting dose of $60 \mathrm{mg}$ at the baseline visit, $25.5 \%$ at a dose

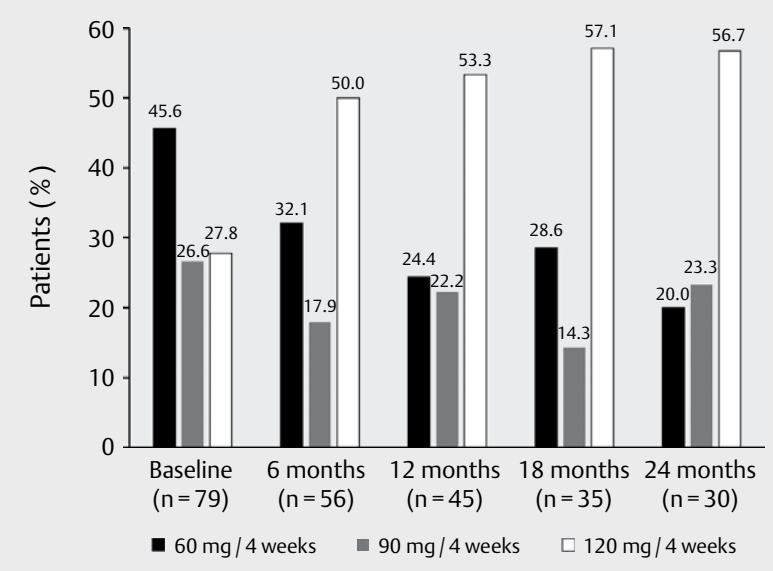

- Fig. 2 LAN doses during the course of the study (efficacy population); LAN: lanreotide autogel.

of $90 \mathrm{mg}$ and $25.5 \%$ at a dose of $120 \mathrm{mg}$ every 4 weeks. The proportion of patients with carcinoid syndrome receiving the $120-\mathrm{mg}$ dose at month 12 was $59.4 \%$ and $68.0 \%$ at month 24 . Throughout the study, the LAN injections were performed in more than $90 \%$ of the patients by a health care professional, in less than $10 \%$ by the patient or her/his partner.

During the study 16 (22.9\%) patients received concomitant NET treatment. Of those, $43.8 \%$ received PRRT, $12.5 \%$ local ablative treatment, $18.8 \%$ chemotherapy and $43.8 \%$ targeted treatment with everolimus $(n=6)$ or sunitinib $(n=1)$ (multiple treatments possible). 


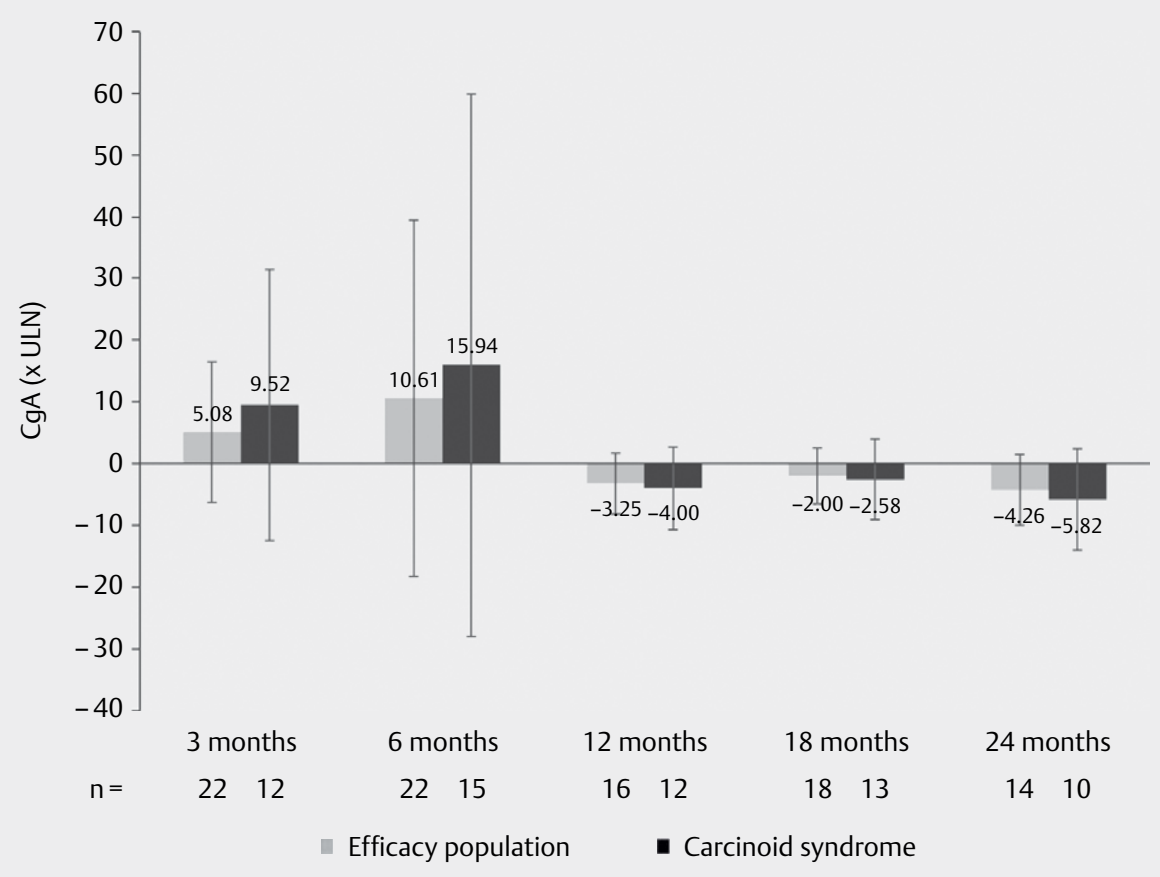

- Fig. 3 Mean change of $\mathrm{CgA}$ values $(95 \% \mathrm{Cl})$ from baseline during the course of the study in the patients of the efficacy population, and post hoc in patients with carcinoid syndrome; mean values at the baseline visit: efficacy population: $13.2 \times \mathrm{ULN}(95 \% \mathrm{Cl}, 0.61-25.81)$; patients with carcinoid syndrome: $21.13 \times$ ULN $(95 \% \mathrm{Cl}, 0.0-42.98)$; CgA: chromogranin A, ULN: upper limit of normal.

\section{Biochemical response}

Effectiveness analyses are presented in the efficacy population. CgA levels were measured to assess long-term biochemical response to LAN treatment. The primary endpoint, disease control at 12 months, defined by a $<50 \%$ CgA increase compared with its lowest value between baseline and month 3, was achieved by 17 of $19(89.5 \%$ [95\% $\mathrm{Cl}, 66.9-98.7])$ patients with evaluable measurements. Since the effects of LAN are mediated by SSTR, biochemical response was evaluated post hoc in the subgroup of 12 patients with positive SSTR scintigraphy at baseline. In this subgroup, the primary endpoint was reached by $83.3 \%(95 \% \mathrm{Cl}, 51.6-97.9)$ patients.

Mean $\mathrm{CgA}(95 \% \mathrm{Cl})$ levels were $13.2 \times$ the upper limit of normal (ULN) (0.6-25.8) at baseline, 24.2 x ULN (0.0-67.7) at 6 months, $2.5 \times \operatorname{ULN}(0.7-4.4)$ at 12 months, and $2.4 \times \operatorname{ULN}(95 \% \mathrm{Cl}, 0.2-4.5)$ at 24 months. CgA levels $>1 \times$ ULN were observed in 27 (71.1\%) patients and 9 (23.7\%) patients had a CgA $>10 \times$ ULN at baseline. In a post hoc analysis in patients with carcinoid syndrome, mean $(95 \% \mathrm{Cl}) \mathrm{CgA}$ levels were $21.1 \times$ ULN $(0.0-43.0)$ at baseline, $33.1 \times$ ULN (0.0-94.8) at month 6, $3.0 \times$ ULN (0.6-5.3) at month 12 , and $2.7 \times$ ULN (-0.3-5.7) at month 24. Due to low numbers, no data are presented for urine levels of 5-HIAA. > Figure 3 shows the mean change of $\mathrm{CgA}$ values between baseline and 3, 6, 12, 18 and 24 months, and in the subgroup of patients with carcinoid syndrome.

\section{Symptoms and QoL, and glucose metabolism}

$38.5 \%$ of patients with carcinoid syndrome reported daily diarrhea episodes at baseline visit ( $70 \%$ of those with 4 or more daily bowel movements), $31.6 \%$ at month 12 and $21.4 \%$ at month 24 ( $\vee$ Fig. $4 a$ ). a

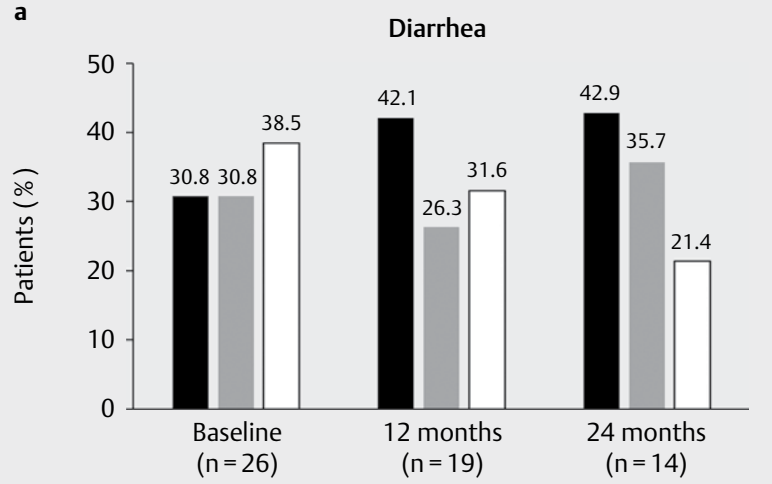

b

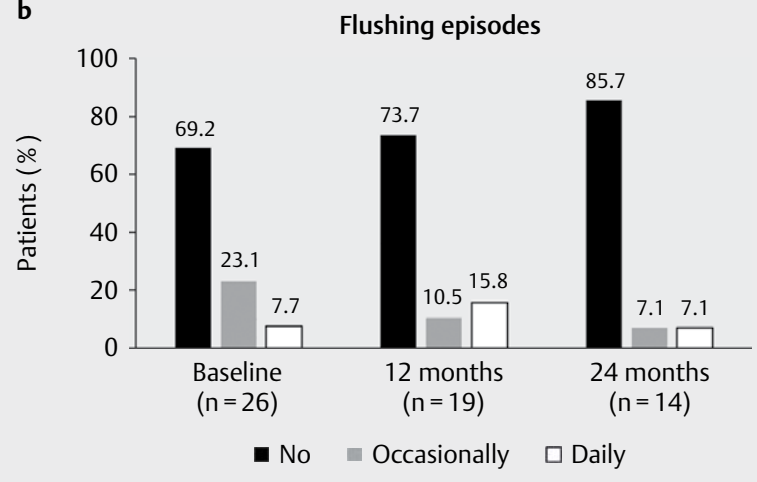

-Fig. 4 Patients with carcinoid syndrome reporting no, occasional or daily a. diarrhea or $\mathbf{b}$. flushing episodes during the course of the study (efficacy population). 
- Table 2 Mean baseline $(95 \% \mathrm{CI})$ and changes from baseline to months 12 and 24 of EORTC QLQ-C30 and disease-specific EORTC GI-NET21 scale scores (efficacy population).

\begin{tabular}{|c|c|c|c|c|c|c|}
\hline & \multicolumn{2}{|c|}{ Baseline } & \multicolumn{4}{|c|}{ Change from baseline } \\
\hline & \multirow[b]{2}{*}{$\mathbf{n}$} & \multirow[b]{2}{*}{ Mean $(95 \% \mathrm{Cl})$} & \multicolumn{2}{|c|}{ Month 12} & \multicolumn{2}{|c|}{ Month 24} \\
\hline & & & $\mathbf{n}$ & Mean (95\% Cl) & $\mathbf{n}$ & Mean $(95 \% \mathrm{Cl})$ \\
\hline \multicolumn{7}{|c|}{ QLQ-C30 global health status/QoL and functional scales ${ }^{\mathrm{a}}$} \\
\hline Global health status & 36 & $54.4(45.6-63.2)$ & 19 & $0.4(-9.5-10.4)$ & 15 & $-5.0(-23.0-13.0)$ \\
\hline Physical functioning & 36 & $72.8(64.3-81.2)$ & 19 & $-2.1(-12.3-8.1)$ & 15 & $-5.8(-18.6-7.0)$ \\
\hline Role functioning & 36 & $60.6(48.9-72.4)$ & 19 & $0.0(-15.6-15.6)$ & 15 & $-3.3(-25.7-19.1)$ \\
\hline Emotional functioning & 7 & $63.2(55.0-71.4)$ & 19 & $-1.2(-13.5-11.2)$ & 15 & $-2.8(-19.5-13.9)$ \\
\hline Cognitive functioning & 36 & $79.2(72.2-86.1)$ & 19 & $-4.4(-15.4-6.6)$ & 15 & $3.3(-9.3-16.0)$ \\
\hline Social functioning & 36 & $64.8(54.0-75.6)$ & 19 & $-6.1(-22.0-9.7)$ & 15 & $-8.9(-33.5-15.7)$ \\
\hline \multicolumn{7}{|l|}{ QLQ - C30 symptom scales ${ }^{b}$} \\
\hline Fatigue & 36 & $45.1(35.1-55.1)$ & 19 & $4.1(-9.9-18.1)$ & 15 & $7.4(-4.1-19.0)$ \\
\hline Nausea and vomiting & 36 & $14.4(6.6-22.1)$ & 19 & $0.0(-11.7-11.7)$ & 15 & $-4.4(-20.6-11.7)$ \\
\hline Pain & 36 & $32.4(21.5-43.3)$ & 19 & $7.9(-11.3-27.1)$ & 15 & $7.8(-12.8-28.4)$ \\
\hline \multicolumn{7}{|l|}{ QLQ-C30 single item scales ${ }^{b}$} \\
\hline Dyspnea & 36 & $31.5(20.7-42.2)$ & 19 & $1.8(-10.8-14.3)$ & 15 & $-2.2(-20.0-15.5)$ \\
\hline Insomnia & 36 & $41.7(29.2-54.1)$ & 19 & $3.5(-18.5-25.5)$ & 15 & $2.2(-21.4-25.8)$ \\
\hline Appetite loss & 35 & $22.9(10.8-34.9)$ & 18 & $-7.4(-25.9-11.1)$ & 14 & $-14.3(-33.8-5.3)$ \\
\hline Constipation & 36 & $2.8(0.0-5.9)$ & 19 & $5.3(-4.4-14.9)$ & 15 & $6.7(-1.0-14.3)$ \\
\hline Diarrhea & 36 & $51.9(37.7-66.0)$ & 19 & $-3.5(-24.9-17.8)$ & 15 & $-6.7(-35.6-22.3)$ \\
\hline Financial difficulties & 35 & $16.2(6.4-26.0)$ & 18 & $7.4(-6.0-20.8)$ & 14 & $7.1(-11.6-25.9)$ \\
\hline \multicolumn{7}{|l|}{ GI-NET21 scales ${ }^{b}$} \\
\hline Endocrine scale & 35 & $21.3(12.2-30.3)$ & 18 & $-7.4(-17.8-3.0)$ & 14 & $-11.9(-25.1-1.3)$ \\
\hline Gastrointestinal scale & 35 & $35.0(28.2-41.7)$ & 18 & $5.6(-7.1-18.2)$ & 14 & $0.5(-10.5-11.4)$ \\
\hline Treatment scale & 21 & $26.7(14.4-39.0)$ & 11 & $1.0(-12.2-14.2)$ & 9 & $11.1(-5.3-27.5)$ \\
\hline Social function scale & 35 & $60.3(51.7-68.9)$ & 18 & $1.9(-13.7-17.4)$ & 14 & $0.8(-15.6-17.1)$ \\
\hline Disease-related worries scale & 35 & $68.1(59.6-76.6)$ & 17 & $-8.5(-23.3-6.3)$ & 14 & $-7.5(-27.6-12.5)$ \\
\hline Muscle/bone pain symptom & 34 & $34.3(22.0-46.6)$ & 18 & $9.3(-10.3-28.8)$ & 14 & $21.4(-2.0-44.8)$ \\
\hline Sexual function & 19 & $50.9(29.2-72.5)$ & 9 & $0.0(-22.2-22.2)$ & 6 & $5.6(-35.3-46.5)$ \\
\hline Information/communication function & 34 & $19.6(10.5-28.7)$ & 17 & $9.8(-10.1-29.7)$ & 12 & $8.3(-20.4-37.1)$ \\
\hline Body image & 35 & $32.4(19.8-45.0)$ & 18 & $-1.9(-22.7-19.0)$ & 14 & $16.7(-6,9-40.2)$ \\
\hline \multicolumn{7}{|c|}{$\begin{array}{l}\text { Score ranges between a. } 0 \text { (worst functioning) and } 100 \text { (best functioning) - higher values indicate better quality of life, and b. between } 0 \text { (no } \\
\text { problems) and } 100 \text { (highest level of problems/symptoms) - lower values indicate better quality of life; table cells highlighted in green indicate } \\
\text { improvement vs. baseline. Green highlighting indicates improvement, red highlighting worsening of quality of life scores vs. baseline of more than } 10 \\
\text { points; Cl: confidence interval; EORTC: European Organisation for Research and Treatment of Cancer; QLQ: quality of life questionnaire; GI-NET: } \\
\text { disease-specific scales for gastrointestinal neuroendocrine tumors. }\end{array}$} \\
\hline
\end{tabular}

At the baseline visit, $30.8 \%$ of patients with carcinoid syndrome experienced occasional or daily flushing episodes, $26.3 \%$ at 12 months and $14.2 \%$ patients at 24 months (>Fig. 4 b).

- Table 2 shows mean $(95 \% \mathrm{Cl}$ ) raw scores and change from baseline to months 12 and months 24 for EORTC QLQ-C30 global health status and functional scales, symptom scales and single item scales as well as of the disease-specific GI-NET21 scales of the efficacy population. Improvements of mean scores of more than 10 points, i. e. changes deemed clinically significant, were observed for the QLQ-C30 single item scale appetite loss as well as for the GI-NET21 endocrine scale at month 24 ( $>$ Table 2). Worsening of more than 10 points was observed for the GI-NET21 treatment, muscle/bone pain symptom and body image scales at month 24 ( $\vee$ Table 2). No clinically significant differences with changes of more 10 points were observed either in the QLQ-C30 global health
status/QoL and functional scales, the QLQ-C30 symptoms scales. However, confidence intervals were wide and the number of patients who completed the questionnaires was low.

Mean $\mathrm{HbA}_{1 \mathrm{c}}$ values were $5.8 \%(95 \% \mathrm{Cl}, 5.5-6.1)$ at baseline, $5.9 \%$ $(95 \% \mathrm{Cl}, 5.7-6.1)$ at month $6,5.9 \%(95 \% \mathrm{Cl}, 5.7-6.1)$ at month 12 , and $7.0 \%(95 \% \mathrm{Cl}, 5.1-8.8)$ at month 24 . The proportion of patients with $\mathrm{HbA}_{1 \mathrm{c}}$ values $<6.5 \%$ remained stable with $\geq 70 \%$ at all visits. Mean fasting glucose was essentially stable in the course of the study (range of mean values: $103.4-111.7 \mathrm{mg} / \mathrm{dL}$ ). The proportion of patients with fasting glucose $<140 \mathrm{mg} / \mathrm{dL}$ was $\geq 80 \%$ at all visits and the proportion of patients who received antidiabetic treatment remained $<20 \%$ for all visits. 


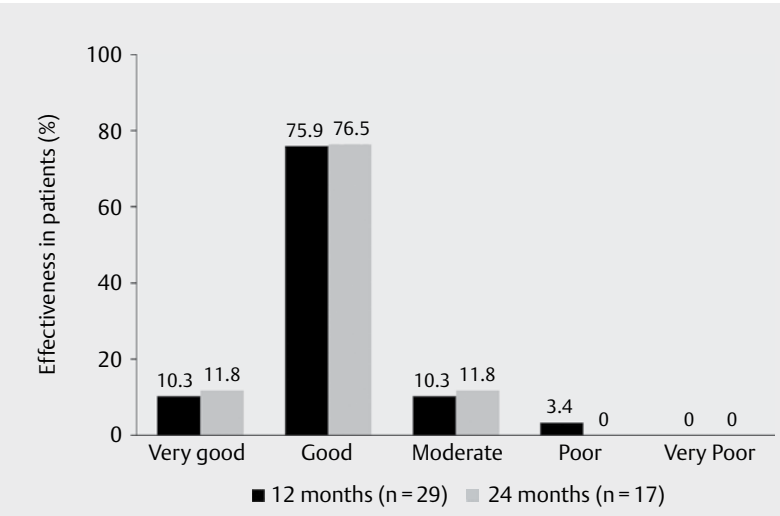

-Fig. 5 Global evaluation of LAN treatment effectiveness by physicians at months 12 and 24; LAN: lanreotide.

\section{RECIST response and overall effectiveness}

At baseline, $7.1 \%$ of patients with available imaging results $(n=14)$ had complete response (CR), $7.1 \%$ partial response (PR), $42.9 \%$ stable disease (SD) and $42.9 \%$ progressive disease (PD). At month $12(n=13)$ no patient had CR or PR, 76.9\% had SD, and $23.1 \%$ had PD. At month $24(n=8) 12.5 \%$ of patients had CR, no patient had PR, $75.0 \%$ had SD and $12.5 \%$ had PD.

Overall effectiveness of treatment with LAN was judged subjectively by the participating physicians as 'very good' or 'good' for $86.2 \%$ of patients at month $12(n=29)$ and for $88.3 \%$ at month 24 $(n=17)$ ( Fig. 5).

\section{Safety and tolerability}

No new safety concerns arose during this study and/or required further investigation. AEs were reported for 43 patients during the study following treatment with LAN. In 18 patients at least one AE was reported as serious, but no serious AEs were deemed 'related' or 'possibly related' to the study medication.

In the enrolled population, at baseline, mean heart rate was 74.4 (95\% Cl, 71.3-77.5) beats per minute (bpm), mean body weight was $73.7(95 \% \mathrm{Cl}, 70.1-77.2) \mathrm{kg}$ and mean systolic and diastolic blood pressures were 136.8 (95\% Cl, $132.2-141.4) \mathrm{mmHg}$ and 78.8 ( $95 \% \mathrm{Cl}, 75.7-81.8) \mathrm{mmHg}$, respectively. During the study, heart rate, body weight and blood pressure remained unchanged. The proportion of patients who received antihypertensive treatment was $50.0 \%$ at baseline and ranged from $37.5 \%$ to $61.5 \%$ between month 3 and month 24.

Ultrasound examinations for gall bladder stones were performed in 49 patients at baseline, in 4 patients at month 1 and in 18 patients at months 6 to 18 during the follow-up. At baseline, gall bladder stones were detected in 4 patients and at month 24 in 7 patients.

Overall tolerability of treatment was judged as 'very good' or 'good' by $94.6 \%$ at month 12 and by $83.3 \%$ of patients at month 24.

\section{Discussion}

Our 24-month observational study of the treatment of NET with LAN in Germany and Austria provides insights into patient characteristics, treatment effectiveness, and disease control in various patient subgroups in a clinical 'real-world' setting. Nearly $90 \%$ of evaluable patients achieved the primary endpoint of a $<50 \%$ increase of $\mathrm{CgA}$ levels at 12 months, which we defined as biochemically stable disease. In addition, imaging showed stable disease according to RECIST1.1 criteria in more than $75 \%$ of the patients at months 12 and 24 . These observations are in line with the known antiproliferative activities of LAN in patients with GEP-NET $[10,11]$. Nevertheless, low sample sizes, missing data and variability of values due to local measurement of biochemical parameters limit the interpretation of our data: target enrolment was exceeded, but fewer patients than expected could be included in the efficacy population. Similarly, the proportion of patients with carcinoid syndrome reporting daily diarrhea at each visit in our post hoc analysis must be interpreted with caution, as different patients may be included at each visit.

Our study population represents patients from two European countries with similar health system characteristics. A median of 1.8 years between onset of symptoms and diagnosis indicates that the NET diagnostic delay was similar between our patient population and that reported in other studies $[16,17]$. With the exception of four patients, all tumors were classified as NET G1/G2 and the vast majority had metastasized. Almost all of the tumors studied immunohistochemically expressed SSTR2/5 and approximately $80 \%$ of patients with NET G1/2 and all patients with NET G3 had a positive SSTR scintigraphy. This is in agreement with literature data on SSTR expression in vitro $[18,19]$ and on reported detection of carcinoid tumors reported from clinical studies $(70-100 \%)[20,21]$. This finding supports current clinical practice not to demand SSTR scintigraphy in order to predict response to somatostatin analogs. With respect to treatment, $90 \%$ of patients had been operated on previously. LAN was the first-line medical treatment being initiated for more than $80 \%$ of our patients. The use of first line somatostatin analogs is in accordance with current German and European guidelines [3,22] generally recommending treatment with somatostatin analogs in patients with advanced GEP-NET G1/G2 as initial antiproliferative or antisecretory therapy.

Within the study period no clinically relevant changes of mean values from baseline to month 24 were observed for 12 of $17 \mathrm{QoL}$ scales assessed including scales for fatigue, pain and diarrhea, which are known to be negatively affected during the course of the disease in patients with NET, especially in those with carcinoid syndrome [23]. On the other hand, clinically relevant improvements of mean values were observed in two QLQ-C30 scales (appetite loss, endocrine scale) and worsening in three GI-NET21 scales (treatment, muscle/bone pain, body image). Still, evaluation of QoL and interpretation of results is difficult in the real-world setting due to the heterogeneity of the clinical presentation of NETs, which is also reflected by the wide confidence intervals.

Overall, participating physicians were satisfied with LAN treatment and rated the overall effectiveness of LAN in almost $90 \%$ of the patients as 'good' or 'very good'. Nevertheless, only a little over half of the patients received the recommended antiproliferative standard LAN dose of $120 \mathrm{mg}$ every 4 weeks at 24 months, sug- 
gesting that there may be potential to further optimize LAN treatment effectiveness in NET patients in a 'real-world' setting. In addition, patients with carcinoid syndrome might also have benefitted from using the full dose range of LAN in order to further reduce diarrhea or flushing episodes. We only can speculate about the reasons for not prescribing the recommended dose $120 \mathrm{mg}$ LAN every 4 weeks in the patients. Some physicians may be worried about side effects, some may prefer individualized dosing and feel satisfied with treatment response to lower dosing. In fact, our data are in line with results of an observational study showing that $50 \%$ of GEP-NET patients treated in the community practice setting received octreotide at a relative dose intensity of less than $85 \%$, $16.7 \%$ received above-label dose [24].

No new safety signals for LAN were observed throughout our study. More than $90 \%$ of the participating patients rated tolerability of LAN as 'good' or 'very good', thus affirming the overall favorable safety profile obtained from controlled studies [5-11].

Limitations of the study are essentially due to the 'real-world' nature of a non-interventional study. All decision-making concerning both diagnosis and treatment was in the hands of the treating physician. Patient population, treatment discontinuations and the number of patients who discontinued and the reduced numbers of valid measurements during the course of the study thus reflect routine clinical practice of the participating centers. Interpretation of results is clearly limited, in particular by a high dropout rate mainly due to decreasing efficacy of treatment and small sample sizes. We had planned to analyse whether early change in $\mathrm{CgA}$ was predictive of long-term treatment success, but the number of evaluable patients was too low. We were also unable to confirm the direct inhibitory action of LAN on excess serotonin production in the enrolled patients with carcinoid syndrome by analyzing urinary 5-HIAA excretion, due to low sample size. This heterogeneity of assessments reflects tailoring of care to individual patients. For example, only a few physicians performed ultrasound examinations during the follow-up visits, in line with guidelines that recommend ultrasound imaging in patients treated with somatostatin analogs at the start of therapy and for monitoring only if clinically indicated $[25,26]$.

In conclusion, this analysis shows favorable effectiveness with stabilization of disease-related symptoms and good tolerability of LAN in clinical practice.

\section{Participating Centers and Principal Investigators}

Department of Gastroenterology and Endocrinology, Philipps-University, Marburg, Germany (Anja Rinke), Hämatologisch-Onkologische Praxis, Würselen, Germany (Christoph Maintz), Praxis für Onkologie Leer-Emden-Papenburg, Leer, Germany (Lothar Müller), Department of Medicine 1, University Medical Center of the Johannes Gutenberg-University Mainz, Mainz, Germany (Matthias Weber), Department of Endocrinology and Metabolism, Division of Laboratory Research, University Hospital Essen, Germany (Harald Lahner), Department of Medicine 1, Division of Endocrinology, Universitätsklinikum Erlangen, Erlangen, Germany (Marianne Pavel), Department of Neuropathology, Pituitary Pathologist, University Hospital Eppendorf, Hamburg, Germany (Wolfgang Säger), ENDOC
Center for Endocrine Tumors, Hamburg, Germany (Stephan Petersenn), Agaplesion Gesellschaft für Klinische Studien mbH, Frankfurt am Main, Germany (Claus Bolling), Klinikum der Johann Wolfgang Goethe-Universität - Medizinische Klinik I, Frankfurt am Main, Germany (Jörg Bojunga), Universitätsklinikum Greifswald - Klinik und Poliklinik für Innere, Greifswald, Germany (Markus Lerch), MVZ Endokrinologikum Berlin, Berlin, Germany (Henrik Biering), Städtische Kliniken Neuss - Chirurgische Klinik I, Neuss, Germany (Peter Goretzki), MVZ Balger Straße - Hämatologie, Internistische Onkologie, Baden-Baden, Germany (Kai Neben), Universitätsklinikum Tübingen - Innere Medizin I, Tübingen, Germany (Michael Bitzer), Onkologisches Versorgungszentrum Friedrichshain - Hämatologie, Onkologie und Palliativmedizin, Berlin, Germany (Herbert Lehahn), Universitätsklinikum Erlangen - Endokrinologie, Diabetes, Erlangen, Germany (Christof Schöfl), Praxis Dres. Etzrodt, Alexopoulos, Etzrodt-Walter, Ulm, Germany (Gwendolin Etzrodt-Walter), Onkologische Schwerpunktpraxis, Kronach, Germany (Martina Stauch), Charité Campus Mitte - Medizinische Poliklinik, Berlin, Germany (Yvonne Dörffel), MVZ Kloster Paradiese, Soest, Germany (Eckhard Böcher), Universitätsklinikum der PMU - Klinik für Innere Medizin III, Salzburg, Austria (Richard Greil), Universitätsklinikum des Saarlandes, Homburg, Germany (Frank Lammert), Medizinische Universität Wien - Universitätsklinik für Innere, Wien, Austria (Markus Raderer), Universitätsklinikum Heidelberg - Innere Medizin I, Heidelberg, Germany (Christian Kasperk), Krankenhaus der Elisabethinen Linz GmbH, Linz, Austria (Karl Aichberger), ODEM Gbr, Lohne, Germany (Matthias Penke), Klinikum Wels-Grieskirchen GmbH, Wels, Austria (Thomas Kühr), Clinical Research Stolberg $\mathrm{GmbH}$, Stolberg, Germany (Matthias Groschek), phase drei - Hämato-Onkologischer Studienkreis am Klinikum, Aschaffenburg, Germany (Manfred Welslau)

Where patient data can be anonymised, Ipsen will share all individual participant data that underlie the results reported in this article with qualified researchers who provide a valid research question. Study documents, such as the study protocol and clinical study report, are not always available. Proposals should be submitted to datasharing@ipsen.com and will be assessed by a scientific review board. Data are available beginning 6 months and ending 5 years after publication; after this time, only raw data may be available.

\section{Acknowledgements}

This study was sponsored by Ipsen. The authors thank all patients involved in the study, as well as their caregivers, care team, investigators and research staff in participating institutions. The authors thank Dr. Philipp Hoffmanns, of Dr. Hoffmanns Gesundheitsberatung $\mathrm{GmbH}$, (formerly an employee of Ipsen) for constructive contributions to the manuscript content, and Dr. Joachim Sauer of DPMedsystems AG, Germany, for providing medical writing support, which was sponsored by Ipsen in accordance with Good Publication Practice guidelines.

\section{Conflict of Interest}

A.R. has received honoraria for presentations by AAA, Falk, IPSEN and Novartis. She also received honoraria for attendance at advisory board 
meetings (Ipsen, Novartis) and travel/congress reimbursements (Ipsen, Novartis). H.L. has received honoraria for presentations by Pfizer, Ipsen and Novartis. He also received honoraria for attendance at advisory board meetings (Pfizer, Ipsen, Novartis) and travel/congress reimbursements (Pfizer, Ipsen, Novartis). C.M. has no COI. S.P. has served as an advisory board member for Ipsen, Crinetics, Takeda, and Novartis, and has received honoraria for speaking at symposia for Ipsen, Novartis, Takeda, and Pfizer. A.H., H.U. are Ipsen employee. L.M. has served as an advisory board member for Ipsen. M.P. received honoraria for presentations by IPSEN, AAA, Novartis, Falk, and Boehringer-Ingelheim, and for advisory role from AAA, IPSEN, Riemser, Lilly, Amgen. W.S. has no COI. M.M.W. has received honoraria as a speaker and consultant from Ipsen and Novartis.

\section{References}

[1] Dasari A, Shen C, Halperin D et al. Trends in the incidence, prevalence, and survival outcomes in patients with neuroendocrine tumors in the United States. JAMA Oncol 2017; 3: 1335-1342

[2] Oberg KE. Gastrointestinal neuroendocrine tumors. Ann Oncol 2010; 21 Suppl 7: vii72-vii80

[3] Pavel M, O'Toole D, Costa F et al. ENETS consensus guidelines update for the management of distant metastatic disease of intestinal, pancreatic, bronchial neuroendocrine neoplasms (NEN) and NEN of unknown primary site. Neuroendocrinology 2016; 103: 172-185

[4] Grozinsky-Glasberg S, Grossman AB, Gross DJ. Carcinoid Heart Disease: From Pathophysiology to Treatment-'Something in the Way It Moves'. Neuroendocrinology 2015; 101: 263-273

[5] Vinik Al, Wolin EM, Liyanage $\mathrm{N}$ et al. Evaluation of lanreotide depot/ Autogel efficacy and safety as a carcinoid syndrome treatment (ELECT): A randomized, double-blind, placebo-controlled trial. Endocr Pract 2016; 22: 1068-1080

[6] O'Toole D, Ducreux M, Bommelaer G et al. Treatment of carcinoid syndrome: A prospective crossover evaluation of lanreotide versus octreotide in terms of efficacy, patient acceptability, and tolerance. Cancer 2000; 88: 770-776

[7] Fisher GA Jr., Wolin EM, Liyanage $N$ et al. Lanreotide therapy in carcinoid syndrome: prospective analysis of patient-reported symptoms in patients responsive to prior octreotide therapy and patients naive to somatostatin analogue therapy in the ELECT phase 3 study. Endocr Pract 2018; 24: 243-255

[8] Ruszniewski P, Valle JW, Lombard-Bohas C et al. Patient-reported outcomes with lanreotide Autogel/Depot for carcinoid syndrome: An international observational study. Dig Liver Dis 2016; 48: 552-558

[9] Ruszniewski P, Ish-Shalom S, Wymenga M et al. Rapid and sustained relief from the symptoms of carcinoid syndrome: Results from an open 6-month study of the 28-day prolonged-release formulation of lanreotide. Neuroendocrinology 2004; 80: 244-251

[10] Caplin ME, Pavel M, Cwikla JB et al. Lanreotide in metastatic enteropancreatic neuroendocrine tumors. N Engl J Med 2014; 371: 224-233

[11] Caplin ME, Pavel M, Cwikla JB et al. Anti-tumour effects of lanreotide for pancreatic and intestinal neuroendocrine tumours: The CLARINET open-label extension study. Endocr Relat Cancer 2016; 23: 191-199
[12] Somatuline Autogel EMA Summary of product characteristics. Ipsen Pharma GmbH, October 2018, https://www.ipsen.com/websites/ Ipsen_Online/wp-content/uploads/sites/21/2019/12/11153003/ Fachinformation_Somatuline-60_90_120-mg-Filmtabletten_StandOkt-2019.pdf

[13] Somatuline Autogel US Prescribing Information. Ipsen Biopharmaceuticals, Inc, June 2019, https://www.ipsen.com/websites/Ipsen_Online/ wp-content/uploads/2019/08/30162316/Somatuline_Depot_Full_Prescribing_Information_7.22.19.pdf

[14] Pavel M, Unger N, Borbath I et al. Safety and QOL in patients with Advanced NET in a phase 3b expanded access study of everolimus. Target Oncol 2016; 11: 667-675

[15] Osoba D, Rodrigues G, Myles J et al. Interpreting the significance of changes in health-related quality-of-life scores. J Clin Oncol 1998; 16: 139-144

[16] Singh S, Granberg D, Wolin E et al. Patient-Reported Burden of a Neuroendocrine Tumor (NET) Diagnosis: Results From the First Global Survey of Patients With NETs. J Glob Oncol 2017; 3: 43-53

[17] Basuroy R, Bouvier C, Ramage JK et al. Presenting symptoms and delay in diagnosis of gastrointestinal and pancreatic neuroendocrine tumours. Neuroendocrinology 2018; 107: 42-49

[18] de Herder WW, Hofland LJ, van der Lely AJ et al. Somatostatin receptors in gastroentero-pancreatic neuroendocrine tumours. Endocr Relat Cancer 2003; 10: 451-458

[19] Reubi JC, Laissue J, Waser B et al. Expression of somatostatin receptors in normal, inflamed, and neoplastic human gastrointestinal tissues. Ann NY Acad Sci 1994; 733: 122-137

[20] Dimitroulopoulos D, Xynopoulos D, Tsamakidis K et al. Scintigraphic detection of carcinoid tumors with a cost effectiveness analysis. World J Gastroenterol 2004; 10: 3628-3633

[21] Oberg K, Eriksson B. Nuclear medicine in the detection, staging and treatment of gastrointestinal carcinoid tumours. Best Pract Res Clin Endocrinol Metab 2005; 19: 265-276

[22] Rinke A, Wiedenmann B, Auernhammer C et al. S2k-Leitlinie Neuroendokrine Tumore, AWMF Register Nr. 021-26. Z Gastroenterol 2018; 56: 583-681

[23] Cella D, Beaumont JL, Hudgens S et al. Relationship between symptoms and health-related quality-of-life benefits in patients with carcinoid syndrome: Post Hoc Analyses From TELESTAR. Clin Ther 2018; 40: 2006-2020.e2002

[24] Jiao X, Pulgar S, Boyd M et al. Treatment patterns and clinical outcomes in patients with metastatic gastroenteropancreatic neuroendocrine tumors treated in the community practice setting in the United States. Pancreas 2018; 47: 173-182

[25] Katznelson L, Laws ER Jr., Melmed S et al. Acromegaly: An endocrine society clinical practice guideline. J Clin Endocrinol Metab 2014; 99 : 3933-3951

[26] Pavel M, Valle JW, Eriksson B et al. ENETS consensus guidelines for the standards of care in neuroendocrine neoplasms: Systemic therapy biotherapy and novel targeted agents. Neuroendocrinology 2017; 105: $266-280$ 